\section{Immunohistochemical analysis of matrix metalloproteinase-13 in human caries dentin}

\author{
C. Loreto, ${ }^{1}$ C. Galanti, ${ }^{2}$ G. Musumeci, ${ }^{1}$ \\ M.C. Rusu, ${ }^{3}$ R. Leonardi ${ }^{2}$ \\ 'Department of Bio-Medical Sciences, \\ Human Anatomy and Histology Section, \\ University of Catania, Italy \\ 2Department of Medical and Surgical \\ Sciences, II Dental Unit, University of \\ Catania, Italy \\ ${ }^{3}$ Discipline of Anatomy, Faculty of Dental \\ Medicine, "Carol Davila" University \\ of Medicine and Pharmacy, Bucharest, \\ Romania
}

\section{Abstract}

The immunoexpression profile of matrix metalloproteinase-13 was investigated for the first time in dentin of human caries and healthy teeth. Twelve permanent premolars (10 caries and 2 sound) were decalcified in ethylenediaminetetraacetic acid and processed for embedding in paraffin wax. Sections 3$4 \mu \mathrm{m}$ in thickness were cut and processed for immunohistochemistry. A mouse monoclonal anti-metalloproteinase-13 antibody was used for localisation using an immunoperoxidase technique. Dentinal immunoreactivity was detected in all teeth; it was weak in sound teeth and strong close to the caries area. These in vivo findings suggest a role for metalloproteinase-13 in the development and progression of adult human dental tissue disorders.

\section{Introduction}

Matrix metalloproteinases (MMPs), collectively known as matrixins, make up a multigene family of 23 zinc-dependent endopeptidases that mediate degradation of virtually all extracellular matrix (ECM) molecules, including native and denatured collagen. ${ }^{1-3}$ They are commonly divided into collagenases, gelatinases, stromelysin, matrilysins, membrane-type MMPs, and others. The biological activities of MMPs can be regulated post-transcriptionally or by interaction with specific MMP tissue inhibitors (TIMPs). ${ }^{4}$ The balance between activated MMPs and their inhibitors determines the extent of ECM remodelling. ${ }^{3}$ MMPs play different roles in the oral environment, ${ }^{3}$ where their activity has been documented in various stages of tissue development and in pathological processes such as periodontal disease, caries, and dental pulp inflammation., ${ }^{1,5-12}$ In particular, mounting evidence indicates that the MMPs found in the dentin matrix or in saliva could be responsible for the dentin organic matrix degradation that follows bacterial acidinduced demineralisation, suggesting an important role for them in caries control and/or progression., ${ }^{513-19}$ Although several MMPs, as far as other important molecules, have been identified in healthy and pathological human dentin and pulp, including caries and inflammation, ${ }^{4,9,18-27}$ data regarding their presence and activity in oral tissues are few, and their precise action remains to be elucidated.

MMP-13 is a collagenase 3 and can degrade ECM components as well as a variety of substrates such as collagen, gelatin, aggrecan, perlecan and fibronectin. ${ }^{4}$ Collagenase expression has been documented in dental pulp and in odontoblasts, ${ }^{4,22}$ in particular a recent work has detected the expression of MMP-13 in pulp of sound and caries teeth, suggesting an important role for it in pulp turnover. ${ }^{4}$ This and a more recent study, ${ }^{28}$ reporting that genetic variations in MMP-13 may contribute to interindividual differences in caries susceptibility, suggested to us that different MMP-13 expression profiles might be found in the two conditions and led us to investigate, for the first time, the immunohistochemical expression of MMP-13 in the dentin of sound and decayed teeth.

\section{Materials and Methods}

\section{Specimen collection}

We studied 12 permanent premolars (2 sound and 10 decayed) that had been extracted at the School of Dentistry, University of Catania (Italy) in view of orthodontic treatment or because of advanced or gross caries, respectively. Sample collection was approved by the local Research Ethics Committee and the informed written consent of each patient was obtained. Exclusion criteria for caries specimens adopted were prior endodontic therapy, any associated dental condition, periapical pathology suggesting the presence of necrotic pulp. Only fully erupted teeth were included and all extractions, which were performed under local anaesthesia (2\% lidocaine 1:80,000 epinephrine), were uncomplicated. Immediately after extraction teeth were placed in saline and fixed in $10 \%$ buffered formalin.

Non-caries specimens selected were teeth displaying no colour change indicating caries in the dentin; those with advanced or gross caries were teeth where the colour change extended through more than half of the dentin thickness.
Correspondence: Dr. Carla Loreto, Department of Bio-Medical Sciences, Anatomy Section, University of Catania, via S. Sofia 87, 95123 Catania, Italy.

Tel. +39.095.3782038 - Fax: +39.095 .3782046 .

E-mail: carla.loreto@unict

Key words: Human teeth, caries, MMP-13, dentin, immunohistochemistry.

Received for publication: 25 September 2013. Accepted for publication: 9 January 2014.

This work is licensed under a Creative Commons Attribution NonCommercial 3.0 License (CC BYNC 3.0).

(C) Copyright C. Loreto et al., 2014

Licensee PAGEPress, Italy

European Journal of Histochemistry 2014; 58:2318 doi:10.4081/ejh.2014.2318

\section{Hard dental tissue preparation for immunohistochemistry}

A groove perpendicular to the long tooth axis was cut with a dental burr equipped with an air/water spray system. Specimens were cut horizontally into halves at the cemento-enamel junction and fixed in 10\% neutral buffered formalin. They were demineralised in ethylenediaminetetraacetic acid (EDTA) decalcification fluid (41.3 g disodium EDTA, $4.4 \mathrm{~g}$ $\mathrm{NaOH}$ in $1000 \mathrm{~mL}$ distilled water) for 6 weeks at $4^{\circ} \mathrm{C}$. After an overnight wash, each half was dehydrated in graded ethanols and processed for embedding in paraffin wax with the anatomical orientation preserved. Sections 3-4 $\mu \mathrm{m}$ in thickness were cut according to routine procedures, mounted on silane-coated slides, and finally air-dried.

\section{Immunohistochemistry}

Samples were processed as previously described ${ }^{29}$ Endogenous peroxidase activity was quenched with $3 \% \mathrm{H}_{2} \mathrm{O}_{2}$ for 10 min. Nonspecific antibody binding was blocked by treatment with normal horse/goat serum diluted 1:20 in phosphate buffered saline (PBS) and $0.1 \%$ bovine serum albumin. Sections were placed in a microwave oven $(750 \mathrm{~W})(5 \mathrm{~min} \times 3)$ in capped polypropylene slide-holders with citrate buffer $(\mathrm{pH} 6.0)$, to unmask antigen sites. They were subsequently incubated with mouse monoclonal antiMMP-13 (anti-collagenase 3) antibody (NeoMarkers, Lab Vision, Fremont, CA, USA) diluted $5-10 \mu \mathrm{l} / \mathrm{mL}$ in PBS overnight at $4^{\circ} \mathrm{C}$. The secondary antibody, biotinylated antimouse/anti-rabbit IgG, was applied for 30 min, followed by avidin-biotin- peroxidase complex (Vector Elite Kit Abbott, Chicago, IL, USA) for $30 \mathrm{~min}$, all at room temperature. The immunoreaction was visualised by incubating 
sections for $4 \mathrm{~min}$ in $0.1 \% 3,3$-diaminobenzidine and $0.02 \%$ hydrogen peroxide solution (DAB Substrate kit, Vector Laboratories, Burlingame, CA, USA). Sections were then lightly counterstained with Mayer's haematoxylin (Histolab Products AB, Göteborg, Sweden) and finally mounted in GVA (glycerol vinyl alcohol aqueous mounting solution) (Zymed, San Francisco, CA, USA).

\section{Positive and negative controls}

Positive controls consisted of breast carcinoma sections; negative controls were tooth sections treated with normal rabbit serum instead of the specific antibodies.

\section{Evaluation of immunohistochemical results}

The staining status was identified as either negative or positive; positive staining was defined as the presence of brown chromogen. MMP-13 staining intensity and the proportion of immunopositive cells were examined independently by three anatomists by light microscopy and recorded. Intensity of staining (IS) was graded independently by three anatomists on a 0 to 4 scale according to the following semiquantitative assessment: $0=$ no detectable staining, 1 , weak staining; 2 , moderate staining; 3, strong staining; 4, very strong staining. The proportion of MMP-13immunopositive cells (extent score=ES) was also evaluated independently by three anatomists and scored as a percentage of the final number of 100 cells into 4 categories: -, $\leq 5 \%$; +, 6-30\%; ++, 31-50\%; +++, $\geq 50 \%$, and $++++=\geq 75 \%$. Counting was performed at 200x magnification. The final staining score (FSS) was the sum of IS and ES.

\section{Statistical analysis}

Data were analysed using the MannWhitney U-test. Significance was set at $\mathrm{P}<0.05$. Mean and standard deviation were calculated for the FSS. Interobserver agreement was expressed as kappa coefficient. All data were analysed with the SPSS program (SPSS ${ }^{\circledR}$ release 16.0, Chicago, IL, USA).

\section{Results}

MMP-13 immunostaining was detected in dentin of both sound and caries teeth with different immunoexpression patterns. Sound dentin exhibited very weak immunoreactivity that was detected only at the peritubular level (ES +; IS: 1) (Figure 1). On the contrary dilated dentinal tubuli close to the caries process showed very strong immunoreactivity (ES ++++ ; IS: 4) both in the peritubular zone and in Tomes' processes (plasma membrane and central area) (Figure 2A-B and 3A-B). MMP-13 immunostaining diminished with increasing distance from the caries process (Figure 2C). Immunoreactivity was not detected in the sections incubated with non-immune serum. The kappa coefficient was 0.89 (almost perfect agreement). The mean FSS was $3.97 \pm 0.52$ (range: 3.7-5.8).

\section{Discussion}

This study shows MMP-13 upregulation in dentin of human caries teeth. In particular, its immunoexpression was weak and confined to the peritubular area in sound dentin and strong at the peritubular level and in Tomes' processes in caries dentin, where it decreased as the distance from the decay process increased.

MMP-13 was first detected in breast cancer and subsequently discovered in a variety of other pathological tissues such as malignant squamous epithelium, chondrosarcoma, and melanoma. ${ }^{30,31}$ MMP-13 expression has been documented in fibroblasts of healing gingival wounds, in temporomandibular joint disc with internal derangement and in developing and remodelled tissues including osteoblasts. ${ }^{32-36}$ In contrast, its expression in normal adult tissue is low or absent. ${ }^{30,31}$ Although collagenase expression has already been described in dental pulp and in odontoblasts, its role in odontoblasts has not been considered important.,22,26 In our opinion the conspicuous MMP-13 immunoexpression found in caries dentin in our study suggests that it could be crucially involved in promoting caries progression due to its ability to degrade many ECM components. ${ }^{4}$ The contribution of MMP-13 to caries lesion progression might thus be related not only to its direct ECM degrading activity but also to its involvement in the activation of other MMPs. ${ }^{31}$

The MMP-13 immunoexpression pattern found in our study, with greater expression being found close to caries lesions, is similar to the one described for other MMPs (e.g., MMP-20, 2, 8, and 9) in crown and root lesions, ${ }^{15,19,27}$ despite often diverse expression levels. ${ }^{27}$ Interestingly, the different immunolabelling of caries and sound teeth documented in our study mimics the expression pattern of TIMP-1 found in human caries dentin in a previous study by our group. ${ }^{37}$ TIMP-1 seems to play a role in curbing hard dental tissue breakdown in the post-injury pathological

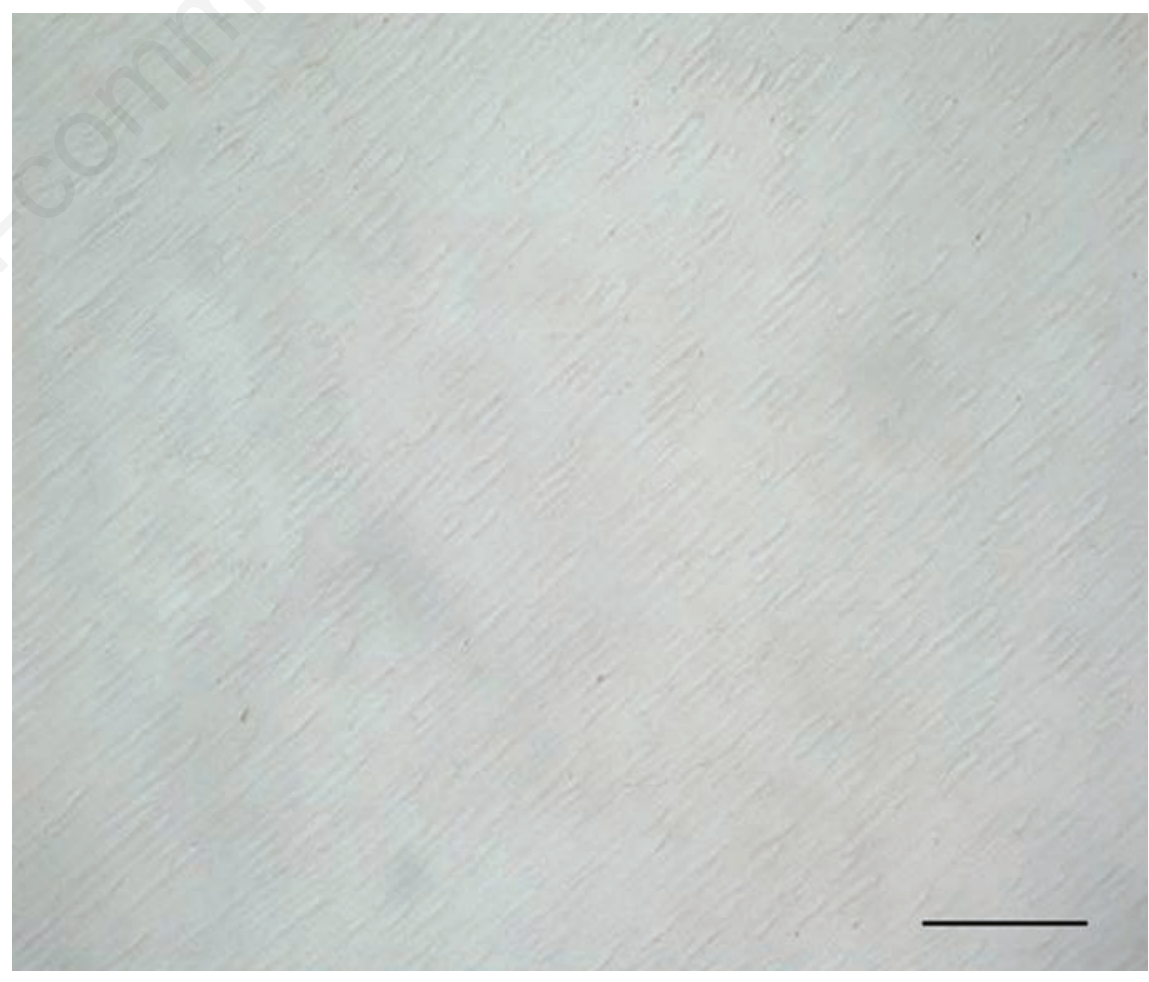

Figure 1. Very weak MMP-13 immunoreaction in sound dentin in the peritubular area. Scale bar: $100 \mu \mathrm{m}$. 
processes taking place in human dental tissues (e.g., caries lesions), ${ }^{11}$ even though the level of TIMPs found in active caries lesions is insufficient to block the progression of dental hard tissue destruction mediated by MMPs, among other agents. ${ }^{36}$ Nonetheless, an important role of MMPs in progression and mainte- nance of the caries process is proved by several recent studies of the effect of synthetic MMP inhibitors, such as doxycycline and chlorhexidine, in reducing collagen degradation in demineralised dentin. ${ }^{38-40}$

In conclusion, our findings suggest a role for MMP-13 in caries. Further research is war- ranted to elucidate the role of MMPs in dentin-pulp complex organisation, pulp pathology, and caries pathogenesis and evolution. Since MMP inhibitors have been shown to slow down the progression of dental caries their utilisation in caries prevention should be further investigated.
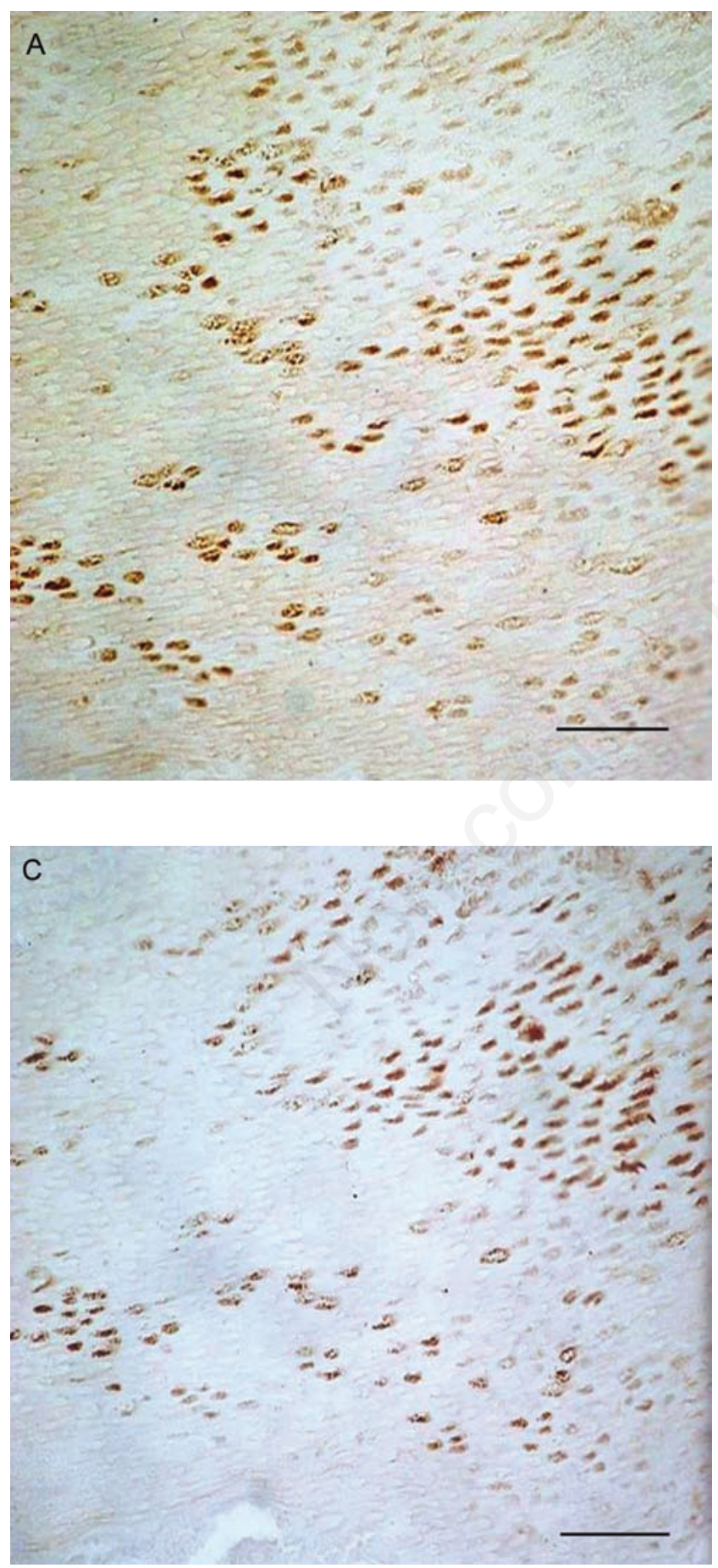

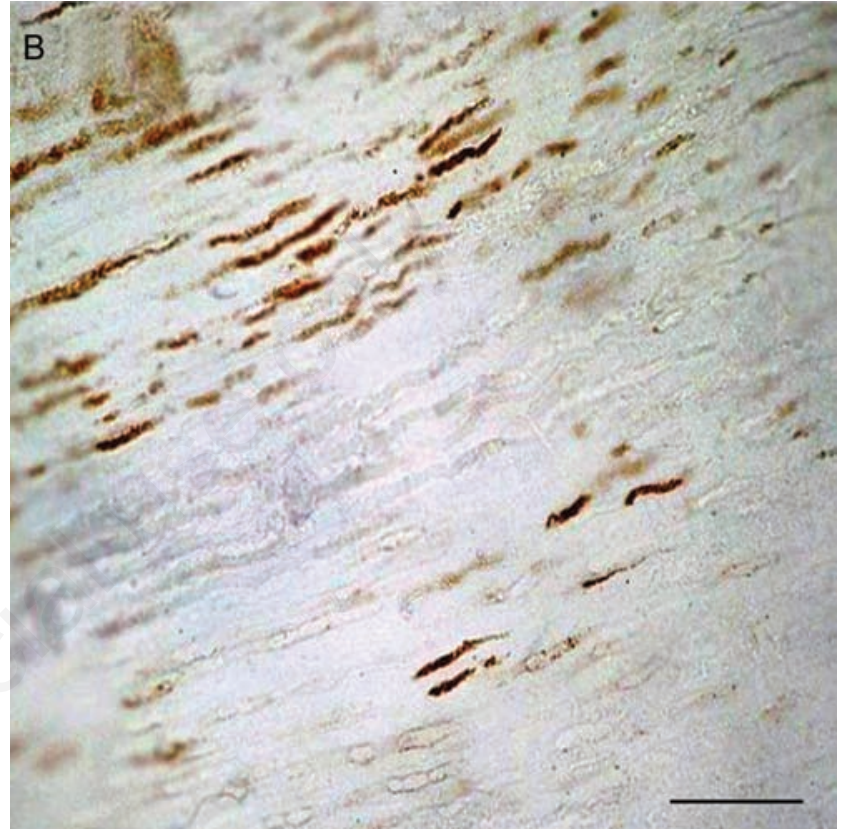

Figure 2. A) Transverse section of a caries tooth with dilated dentinal tubuli displaying very strong MMP-13 immunoreaction in the peritubular area and in Tomes' processes (black arrow); B) Longitudinal section of a caries tooth with dilated dentinal tubuli exhibiting very strong MMP-13 immunoreaction in Tomes' processes (plasma membrane and central area ) (black arrow). C) Transverse section of a caries tooth with very strong MMP-13 immunostaining in the peritubular area (black arrow) that decrease with the increasing distance (asterisk) from the caries lesion. Scale bars: $100 \mu \mathrm{m}$. 

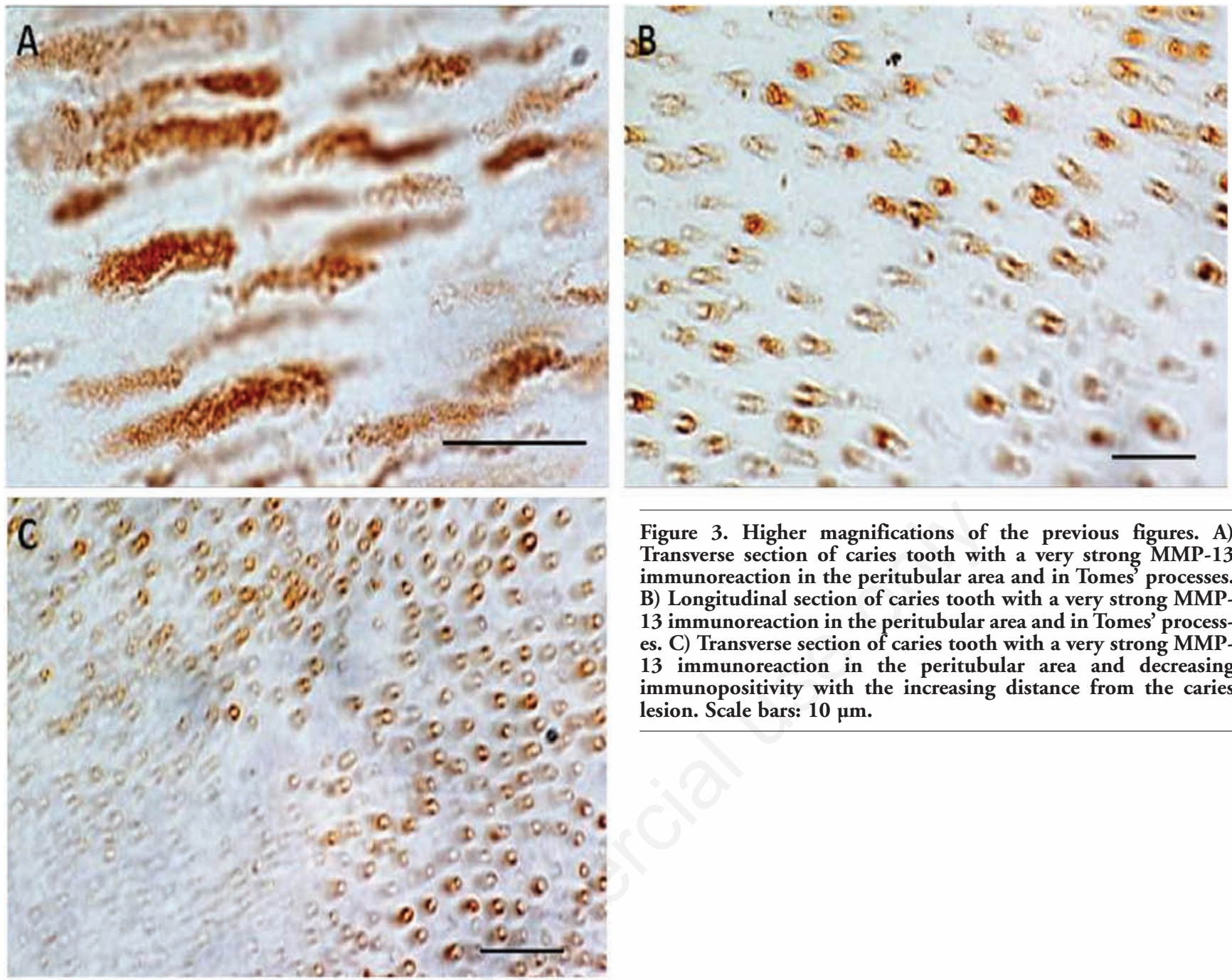

Figure 3. Higher magnifications of the previous figures. A) Transverse section of caries tooth with a very strong MMP-13 immunoreaction in the peritubular area and in Tomes' processes. B) Longitudinal section of caries tooth with a very strong MMP13 immunoreaction in the peritubular area and in Tomes' processes. C) Transverse section of caries tooth with a very strong MMP13 immunoreaction in the peritubular area and decreasing immunopositivity with the increasing distance from the caries lesion. Scale bars: $10 \mu \mathrm{m}$.

\section{References}

1. Birkedal-Hansen H. Role of matrix metalloproteinases in human periodontal diseases. J Periodontol 1993;64:474-84.

2. Visse R, Nagase H. Matrix metalloproteinases and tissue inhibitors of metalloproteinases: structure, function, and biochemistry. Circ Res 2003;92:827-39.

3. Hannas AR, Pereira JC, Granjeiro JM, Tjäderhane L. The role of matrix metalloproteinases in the oral environment. Acta Odontol Scand 2007;65:1-13.

4. Palosaari H , Pennington CJ, Larmas M, Edwards DR, Tjäderhane L, Salo T. Expression profile of matrix metalloproteinases (MMPs) and tissue inhibitors of MMPs in mature human odontoblasts and pulp tissue. Eur J Oral Sci 2003;111:117-27.

5. Ingman $\mathrm{T}$, Tervahartiala $\mathrm{T}$, Ding $\mathrm{Y}$, Tschesche H, Haerian A, Kinane DF, et al. Matrix metalloproteinases and their inhibitors in gingival crevicular fluid and saliva of periodontitis patients. J Clin Periodontol 1996;23:1127-32.

6. Borsani E, Salgarello S, Mensi M, Boninsegna R, Stacchiotti A, Rezzani R, et al. Histochemical and immunohistochemical evaluation of gingival collagen and metalloproteinases in peri-implantitis. Acta Histochem 2005;107:231-40.

7. Leonardi R, Caltabiano R, Loreto C. Collagenase-3 (MMP-13) is expressed in periapical lesions: an immunohistochemical study. Int Endod J 2005;38:297-301.

8. Beklen A, Tüter G, Sorsa T, Hanemaaijer R, Virtanen I, Tervahartiala T, et al. Gingival tissue and crevicular fluid co-operation in adult periodontitis. J Dent Res 2006;85: 59-63.

9. Chaussain-Miller C, Fioretti F, Goldberg M, Menashi S. The role of matrix metalloproteinases (MMPs) in human caries. J Dent Res 2006;85:22-32.

10. Leonardi R, Talic NF, Loreto C. MMP-13 (collagenase 3) immunolocalisation during initial orthodontic tooth movement in rats. Acta Histochem 2007;109:215-20.

11. Leonardi R, Matthews JB, Caltabiano R, Greco $\mathrm{M}$, Lombardo $\mathrm{C}$, Loreto $\mathrm{C}$, et al. MMP-13 expression in keratocyst odontogenic tumour associated with NBCCS and sporadic keratocysts. Oral Dis 2010;16: 795-800.

12. Leonardi R, Loreto C, Barbato E, Caltabiano R, Lombardo C, Musumeci G, et al. MMP-13 (collagenase 3) localization in human temporomandibular joint discs with internal derangement. Acta Histochem 2008;110:314-8.

13. Dayan D, Binderman I, Mechanic GL. A preliminary study of activation of collagenase in carious human dentine matrix. Arch Oral Biol 1983;28:185-7.

14. Martin-De Las Heras S, Valenzuela A, Overall CM. The matrix metalloproteinase gelatinase A in human dentine. Arch Oral Biol 2000;45:757-65. 
15. Sulkala M, Larmas M, Sorsa T, Salo T, Tjäderhane L. The localization of matrix metalloproteinase-20 (MMP-20, enamelysin) in mature human teeth. J Dent Res 2002;81:603-7.

16. Pashley DH, Tay FR, Yiu C, Hashimoto M, Breschi L, Carvalho RM, et al. Collagen degradation by host-derived enzymes during aging. J Dent Res 2004;83:216-21.

17. Sulkala M, Tervahartiala T, Sorsa T, Larmas M, Salo T, Tjäderhane L. Matrix metalloproteinase-8 (MMP-8) is the major collagenase in human dentin. Arch Oral Biol 2007;52:121-7.

18. Tjäderhane L, Salo T, Larjava H, Larmas M, Overall CM. A novel organ culture method to study the function of human odontoblasts in vitro: gelatinase expression by odontoblasts is differentially regulated by TGF-betal. J Dent Res 1998;77:1486-96.

19. Toledano M, Nieto-Aguilar R, Osorio R, Campos A, Osorio E, Tay FR, et al. Differential expression of matrix metalloproteinase-2 in human coronal and radicular sound and carious dentine. J Dent 2010;38:635-40.

20. Martini D, Trirè A, L Breschi, Mazzoni A, Teti G, Falconi M, Ruggeri A Jr. Dentin matrix protein 1 and dentin sialophosphoprotein in human sound and carious teeth: an immunohistochemical and colorimetric assay. Eur J Histochem 2013;57:e32.

21. Teti G, Salvatore V, Ruggeri A, Manzoli L, Gesi M, Orsini G, Falconi M. In vitro reparative dentin: a biochemical and morphological study. Eur J Histochem 2013;57: e23.

22. Palosaari H, Wahlgren J, Larmas M, Rönkä $\mathrm{H}$, Sorsa T, Salo T, et al. The expression of MMP-8 in human odontoblasts and dental pulp cells is down-regulated by TGF-betal. J Dent Res 2000;79:77-84.

23. Niu LN, Zhang L, Jiao K, Li F, Ding YX, Wang DY, et al. Localization of MMP-2, MMP-9, TIMP-1, and TIMP-2 in human coronal dentine. J Dent 2011;39:536-42.
24. Tjäderhane L, Larjava H, Sorsa T, Uitto VJ, Larmas M, Salo T. The activation and function of host matrix metalloproteinases in dentin matrix breakdown in caries lesions. J Dent Res 1998;77:1622-9.

25. Gusman H, Santana RB, Zehnder M. Matrix metalloproteinase levels and gelatinolytic activity in clinically healthy and inflamed human dental pulps. Eur J Oral Sci 2002;110:353-7.

26. Sulkala M, Pääkkönen V, Larmas M, Salo T, Tjäderhane L. Matrix metalloproteinase-13 (MMP-13, collagenase-3) is highly expressed in human tooth pulp. Connect Tissue Res 2004;45:231-7.

27. Shimada Y, Ichinose S, Sadr A, Burrow MF, Tagami J. Localization of matrix metalloproteinases (MMPs-2, 8, 9 and 20) in normal and carious dentine. Aust Dent $\mathrm{J}$ 2009;54:347-54.

28. Tannure PN, Küchler EC, Falagan-Lotsch P, Amorim LM, Raggio Luiz R, Costa MC, et al. MMP13 polymorphism decreases risk for dental caries. Caries Res 2012;46:4017.

29. Loreto C, Lo Castro E, Musumeci G, Loreto F, Rapisarda G, Rezzani R, et al. Aquaporin 1 expression in human temporomandibular disc. Acta Histochem 2012; 114: 744-48.

30. Freije JM, Díez-Itza I, Balbín M, Sánchez LM, Blasco R, Tolivia J, et al. Molecular cloning and expression of collagenase- 3 , a novel human matrix metalloproteinase produced by breast carcinomas. J Biol Chem 1994;269:16766-73.

31. Leeman MF, Curran S, Murray GI. The structure, regulation, and function of human matrix metalloproteinase-13. Crit Rev Biochem Mol Biol 2002;37:149-66.

32. Ravanti L, Häkkinen L, Larjava $\mathrm{H}$, Saarialho-Kere U, Foschi M, Han J, et al. Transforming growth factor-beta induces collagenase-3 expression by human gingival fibroblasts via p38 mitogen-activated protein kinase. J Biol Chem 1999;274: 37292-300.
33. Reboul P, Pelletier JP, Tardif G, Cloutier JM, Martel-Pelletier J. The new collagenase, collagenase- 3 , is expressed and synthesized by human chondrocytes but not by synoviocytes. A role in osteoarthritis. $\mathrm{J}$ Clin Invest 1996;97:2011-9.

34. Johansson N, Saarialho-Kere U, Airola K, Herva R, Nissinen L, Westermarck J, et al. Collagenase-3 (MMP-13) is expressed by hypertrophic chondrocytes, periosteal cells, and osteoblasts during human fetal bone development. Dev Dyn 1997;208:38797.

35. Ståhle-Bäckdahl M, Sandstedt B, Bruce K, Lindahl A, Jiménez MG, Vega JA, et al. Collagenase-3 (MMP-13) is expressed during human fetal ossification and reexpressed in postnatal bone remodeling and in rheumatoid arthritis. Lab Invest 1997;76:717-28

36. Loreto C, Leonardi R, Musumeci G, Pannone G, Castorina S. An ex vivo study on immunohistochemical localization of MMP-7 and MMP-9 in temporomandibular joint discs with internal derangement. Eur J Histochem 2013;57:e12.

37. Leonardi R, Loreto C. Immunohistochemical localization of tissue inhibitor of matrix metalloproteinase-1 (TIMP-1) in human carious dentine. Acta Histochem 2010;112:298-302.

38. Osorio R, Yamauti M, Osorio E, RuizRequena ME, Pashley DH, Tay FR, et al. Zinc reduces collagen degradation in demineralized human dentin explants. J Dent 2011;39:148-53.

39. Toledano M, Yamauti M, Osorio E, Osorio R. Zinc-inhibited MMP-mediated collagen degradation after different dentine demineralization procedures. Caries Res 2012;46:201-7.

40. Zheng X, Hu J, Chen Y, Zhu Y, Chen H. AFM study of the effects of collagenase and its inhibitors on dentine collagen fibrils. $\mathrm{J}$ Dent 2012;40:163-71. 